Classification

Physics Abstracts

$61.30 \mathrm{G}-64.70 \mathrm{M}-77.40$

\title{
New dielectric relaxation in a bilayered fluid smectic phase
}

\author{
L. Benguigui
}

Solid State Institute, Technion, Israel Institute of Technology, 32000 Haifa, Israel

and F. Hardouin

Centre de Recherches Paul Pascal, Domaine Universitaire, Université de Bordeaux I, 33405 Talence, France

(Reçu le 21 octobre 1983, révisé le 7 décembre, accepté le 20 décembre 1983)

\begin{abstract}
Résumé. - Nous présentons des mesures diélectriques effectuées sur le composé cyané " $\mathrm{DB}_{8} \mathrm{Cl}$ » qui présente la séquence de phases smectiques fluides bicouches : Smectique $A_{2} \rightarrow$ Smectique $C_{2} \rightarrow$ Smectique ?. Nous observons en particulier une nouvelle relaxation perpendiculaire en phase $S_{\text {? }}$, qui vraisemblablement traduit un ordre de dipôles. Un modèle simplifié rendant compte de ce résultat est proposé.

Abstract. - We present dielectric measurements of the " $\mathrm{DB}_{8} \mathrm{Cl}$ » cyano compound which exhibits a Smectic $\mathrm{A}_{2} \rightarrow$ Smectic $\mathrm{C}_{2} \rightarrow$ Smectic ? sequence of bilayered fluid smectic phases. In particular a new perpendicular relaxation is observed in the $S_{r}$ which we interpret as indicative of a dipole ordering. A simplified model taking this result into account is proposed.
\end{abstract}

\section{Introduction.}

Recently a new smectic phase in the compound octyl-phenyl 2-chloro-4-(p-cyano-benzoyloxy) benzoate ( $\mathrm{DB}_{8} \mathrm{Cl}$ » for short) [1] was discovered. Below the nematic state there first appear a smectic $\mathrm{A}_{2}$ phase with a commensurate bilayer structure, followed, when the temperature is decreased by a smectic $\mathrm{C}_{2}$, tilted phase in which the bilayer structure is kept. At lower temperatures the new smectic phase (smectic ?) exists over the interval $107^{\circ} \mathrm{C}-85^{\circ} \mathrm{C}$.

No structural change between this phase and the $\mathrm{S}_{\mathrm{C}_{2}}$ is observed on the X-ray patterns. Both phases exhibit a bilayer structure (as $S_{A_{2}}$ ) and the molecular order inside the layers remains liquid-like. The only noticeable difference between the $S_{\text {? }}$ and the $S_{C_{2}}$ phases is that the 001 Bragg spots become more intense and more numerous in $S_{7}$. It is possible that there is no qualitative modification between these two phases but before coming to this conclusion it is necessary to study their physical properties.

Let us note that similar polar systems exhibit $S_{A}-S_{A}$ transitions [2] and that dipole ordering has been observed with particular dielectric properties in some of these $S_{A}$ phases $[3,4]$. This is why we have measured the dielectric properties of the $« \mathrm{DB}_{8} \mathrm{Cl} »$ compound and in fact, our results give support of a dipole ordering characteristic of the $S_{\text {? }}$ phase. 


\section{Experimental results.}

The real and imaginary parts of the dielectric constant $\varepsilon^{*}=\varepsilon^{\prime}-j \varepsilon^{\prime \prime}$ were measured from $5 \times 10^{3} \mathrm{~Hz}$ to $1 \mathrm{GHz}$ by several methods : Bridge, $Q$ meter and reflectometry, in the directions parallel $\left(\varepsilon_{\|}\right)$ and perpendicular $\left(\varepsilon_{\perp}\right)$ to the director. The sample was oriented by a magnetic field of $13 \mathrm{kG}$.

On figure 1, we present the thermal variations of $\varepsilon_{\|}$and $\varepsilon_{\perp}$ (real part of the complex dielectric constant) at $5 \mathrm{kHz}$ over the whole mesomorphic range. This frequency is below most of the frequencies of absorption domains and higher than frequencies of the conductivity mechanism; thus, the measured values are not too far from the quasi static ones. $\varepsilon_{\|}$decreases as the temperature decreases and $\varepsilon_{\perp}$ varies slowly around 3.7. The transitions are marked only by small anomalies. The relatively low values of $\varepsilon_{\|}$and $\varepsilon_{\perp}$ are indicative of an antiferroelectric ordering.

In this respect $\mathrm{DB}_{8} \mathrm{Cl}$ is quite analogous to the $\mathrm{DB}_{6}$ compound which has only a $\mathrm{S}_{\mathrm{A}_{2}}$ phase $[3,4]$. The results concerning the relaxations are more interesting.

One of the authors [5] has recently predicted that in the $S_{A_{2}}$ phase, due to the long range A.F. ordering, we should observe a second parallel relaxation with the amplitude proportional to $\left(T_{\mathrm{NS}}-T\right)^{2}$ where $T_{\mathrm{NS}}$ is the temperature of the nematic-smectic $\mathrm{A}_{2}$ transition. On figure 2, we

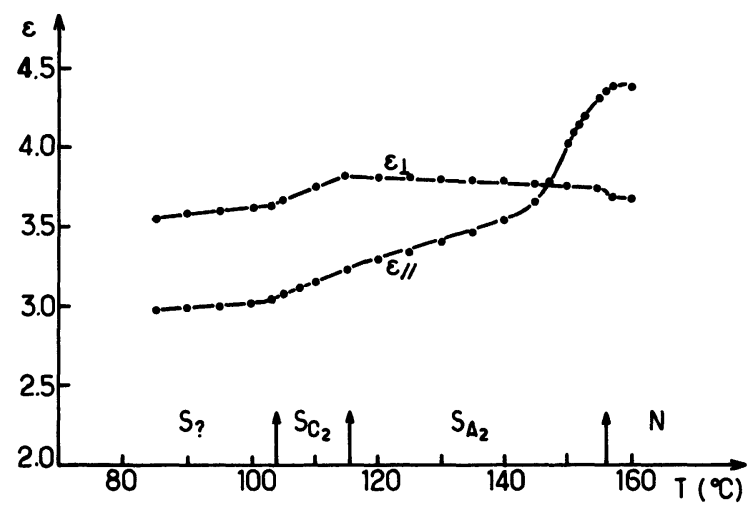

Fig. 1. - Thermal variations of the quasi static dielectric constants $\varepsilon_{\|}$and $\varepsilon_{\perp}$.

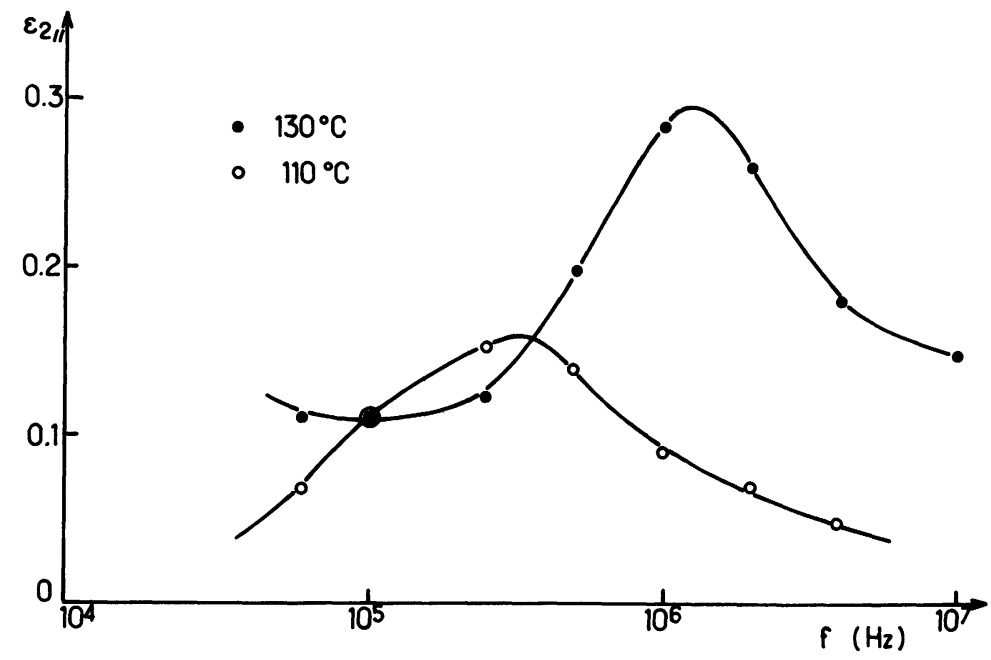

Fig. 2. - Variations of $\varepsilon_{2 \|}$ as function of the frequency showing the existence of a distortion at $110^{\circ} \mathrm{C}$. 
show $\varepsilon_{2 \|}$, the imaginary part of the dielectric constant, as a function of the frequency at $130^{\circ} \mathrm{C}$ $\left(\mathrm{S}_{\mathrm{A}_{2}}\right)$ and $110^{\circ} \mathrm{C}\left(\mathrm{S}_{\mathrm{C}_{2}}\right)$. At $110^{\circ} \mathrm{C}$ the diagram shows a distortion. If this distortion is assumed to be the result of the superposition of two absorption domains, this is in qualitative agreement with the predictions at low temperature.

However, the most striking feature concerns the dynamic permittivity when the electric field is perpendicular to the director. In figure 3 the variation of $\varepsilon_{2 \perp}$ is plotted as a function of $f$ at different temperatures. In the nematic, $S_{\mathrm{A}_{2}}$ and $\mathrm{S}_{\mathrm{C}_{2}}$ phases, we observe one relaxation around $280 \mathrm{MHz}$. This is the usual relaxation associated with the rotation around the long molecular axis, and as expected it is almost independent of $T$. But in the $S_{\text {? }}$ phase, an unexpected second relaxation appears around 10-20 $\mathrm{MHz}$. The thermal dependence of the corresponding relaxation frequency is weak, while the amplitude increases as $T$ decreases. We interpret this last property as indicative of a dipole ordering in the $S_{\text {? }}$ phase. Nevertheless, the measurement by itself does not give any indication of what kind of ordering corresponds to this extra relaxation. We present a model based on energy arguments which permits us to explain the known structural properties of the $S_{\text {? }}$ phase.

\section{Tentative model.}

We can envision that in a perfect A.F. ordering of the $S_{\mathrm{A}_{2}}$ phase, the end dipoles of the molecules in one single-layer are in the same direction (say up) and in the following single-layer all the end dipoles are in the opposite direction (down). The two single-layers form the basic fluid bilayer from which we get 001 Bragg reflection at $d \simeq 2 l$ [6]. At short range we can consider that in each monolayer the molecules are (on average) on a triangular lattice. In such an arrangement the dipolar interaction energy is minimized by the interpenetration of the end dipoles and this should be the qualitative explanation of the $\mathrm{S}_{\mathrm{A}_{2}}$ structure. It is possible to show that when the molecules are tilted, the dipole-dipole interaction is increased. In other words the A.F. ordering of the end dipoles competes with the mechanism favouring the appearance of the $S_{C}$ phase. When the temperature decreases, the competition becomes more severe and it may happen that the system prefers to adopt a new structure with a lower energy. This is a possible explanation for the $S_{\text {? }}$ formation.

Since our argument is only qualitative and based on energy considerations (neglecting the entropy) we shall present a calculation of the dipole-dipole energy of a row of point dipoles instead

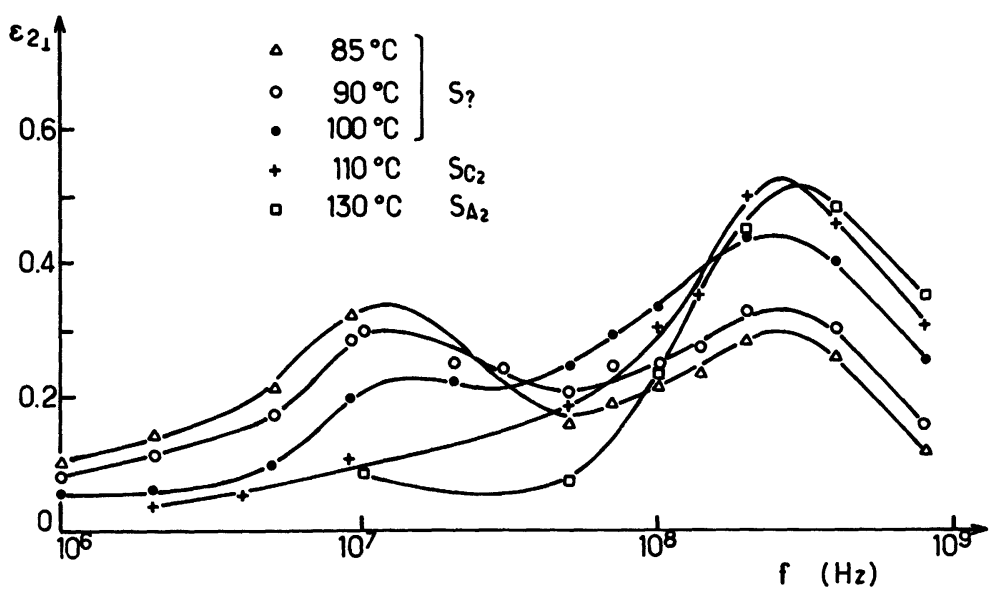

Fig. 3. - Variations of $\varepsilon_{2 \perp}$ versus the frequency. The $S_{\text {? }}$ phase is characterized by a new perpendicular relaxation. 
of considering the two-dimensional array of end dipoles belonging to the two consecutive and interpenetrating single layers. The calculations have only an illustrative interest but the conclusions drawn from the linear model are qualitatively identical to those drawn from a complete twodimensional calculation.

Let us consider a line of point dipoles $\mu$ equally spaced with distance $a$ between them. In the situation analogous to the $\mathrm{S}_{\mathrm{A}_{2}}$ phase, the dipoles are directed perpendicularly to the row (Fig. 4). The interaction energy of $N$ dipoles is :

$$
\begin{aligned}
& U_{\mathrm{A}_{2}}=-\frac{N \mu^{2}}{2 a^{3}}\left(1-\frac{1}{2^{3}}+\frac{1}{3^{3}}-\frac{1}{4^{3}}+\cdots\right) \\
& U_{\mathrm{A}_{2}}=-0.9 \frac{N \mu^{2}}{2 a^{3}} .
\end{aligned}
$$

In the case of the $S_{C_{2}}$ phase, the dipoles make an angle $\alpha$ with the normal to the row (Fig. 4). The dipole-dipole energy is given by :

$$
\begin{aligned}
& U_{\mathrm{C}_{2}}=-\frac{N \mu^{2}}{2 a^{3}}\left(1-3 \sin ^{2} \alpha\right)\left(1-\frac{1}{2^{3}}+\frac{1}{3^{3}} \cdots\right) \\
& U_{\mathrm{C}_{2}}=-0.9 \frac{N \mu^{2}}{2 a^{3}}\left(1-3 \sin ^{2} \alpha\right)
\end{aligned}
$$

$U_{\mathrm{C}_{2}}$ is larger than $U_{\mathrm{A}_{2}}$ : this shows the competition between the two orderings.

Since we observed a new relaxation in the $S_{\text {? }}$ when the field is perpendicular to the director, we have a new contribution to the molecular dipole moment in this direction. This contribution increases when $T$ decreases. Thus our suggestion for the $S_{\text {? }}$ structure is the following : the tilt angle of two consecutive monolayers will not be equal to $\alpha$ as in the $S_{C_{2}}$ phase, but slightly different : $(\alpha+\beta)$ in one monolayer and $(\alpha-\beta)$ in the next one (see Fig. 4). As $\alpha$ is the order parameter of
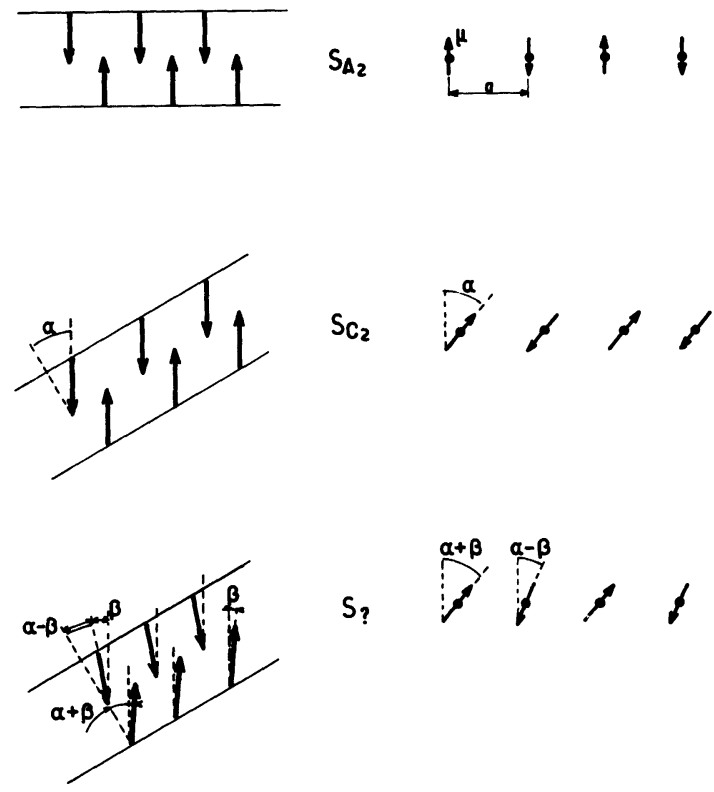

Fig. 4. - Proposed structure of the $S_{A_{2}}, S_{C_{2}}$ and $S_{\text {? }}$ phases and row of dipoles corresponding to each phase. 
the $\mathrm{S}_{\mathrm{C}_{2}}$ phase, $\beta$ can be seen as the order parameter of the $\mathrm{S}_{\text {? }}$ one. In our linear model we alternatively have dipoles making angle $(\alpha+\beta)$ and $(\alpha-\beta)$ with the normal. The dipole-dipole interaction energy of the row of dipoles is now :

$$
U_{\mathrm{S}_{?}}=-0.9 \frac{N \mu^{2}}{2 a^{3}}\left(1-3 \sin ^{2} \alpha\right)-1.05 \frac{N \mu^{2}}{2 \dot{a}^{3}} \sin ^{2} \beta \text {. }
$$

The energy in this structure is less than in the structure for which all the dipoles make the same angle with the normal. Therefore this mechanism may explain the formation of the $S_{\text {? }}$. In the $S_{A_{2}}$ and $S_{C_{2}}$, the end dipole contributes only to the parallel dipole moment (contribution to $\varepsilon_{\|}$), but in our $S_{\text {? }}$ model it contributes also to $\varepsilon_{\perp}$. In the linear approach, the extra dipole moment is equal to $\mu \sin \beta$.

The model can explain - $-a)$ The new relaxation perpendicular to the long molecular axis. - b) The fact that the layers of the $S_{\text {? }}$ are well defined since the permeation (passage of molecules from one layer to the next one) is hindered. Consequently the X-ray reflection orders on the layers are more intense and numerous than in the $\mathrm{S}_{\mathrm{A}_{2}}$ or $\mathrm{S}_{\mathrm{C}_{2}}$ phases. $-c$ ) The asymmetric bimolecular shape in $S_{\text {? }}$ conjectured from the $X$-ray analysis of the intensity profile of the diffuse spot at wide angles [1].

\section{Conclusion.}

The dielectric measurements of $\mathrm{DB}_{8} \mathrm{Cl}$ show that the $\mathrm{S}_{\text {? }}$ phase is really qualitatively different from the $S_{C_{2}}$ phase : in $S_{\text {? }}$ a new relaxation appears around $10-20 \mathrm{MHz}$ in the perpendicular configuration. We interpret it as the indication of a dipole ordering. Starting from simplified energetic arguments we describe a tentative model for the $S_{\text {? }}$ structure. More detailed calculations and experimental evidences are needed to put the model on a firm basis.

\section{References}

[1] Hardouin, F., Nguyen Huu Tinh, Levelut, A. M., J. Physique Lett. 43 (1982) L-779.

[2] Hardouin, F., Levelut, A. M., Achard, M. F., Sigaud, G., J. Chim. Phys. 80 (1983) 53 and references therein.

[3] Benguigui, L., Hardouin, F., J. Physique Lett. 42 (1981) L-381.

[4] Druon, C., Wacrenier, J. M., Hardouin, F., Nguyen Huu Tinh, Gasparoux, H., J. Physique 44 (1983) 1195.

[5] Benguigui, L., J. Physique 44 (1983) 273.

[6] Hardouin, F., Levelut, A. M., Benattar, J. J., Sigaud, G., Solid State Commun. 33 (1980) 337. 